\title{
Pseudomonas diarrhea in a child suffering from acute lymphatic leukemia
}

A. De, H. Mathurkar,

S. Baveja,

M. V. Manglani ${ }^{1}$

Department of Microbiology and ${ }^{1}$ Pediatrics,

L.T.M. Medical College,

Sion, Mumbai, India

\section{Address for correspondence:}

Dr. Anuradha De,

Department of Microbiology,

L.T.M. Medical College,

Sion, Mumbai - 400 022, India.

E-mail: dr_anuradhade@yahoo.com

\section{A B S T R A C T}

\begin{abstract}
A female child admitted to hospital, diagnosed with acute lymphatic leukemia - CALLA positive, developed loose motions. Her stool culture and blood culture grew Pseudomonas aeruginosa. Although the diarrhea subsided after five days, the stool culture repeatedly grew $P$. aeruginosa for more than one month, in spite of treatment. Even though diarrhea due to Pseudomonas is rare, it can yet be seen in immunocompromised patients and is also associated with neutropenic enterocolitis. Stool specimens of all leukemia patients on chemotherapy and suffering from diarrhea, should be sent routinely for culture, so as to find out the exact cause of the diarrhea. Proper reporting will enable the clinicians to start appropriate antibiotics, thereby, reducing the morbidity and mortality of the leukemia patients.
\end{abstract}

Key words: Acute lymphatic leukemia, Pseudomonas, diarrhea

DOI: $10.4103 / 0971-5851.65341$

\section{INTRODUCTION}

Pseudomonas is notorious for causing hospital-associated infections, infection in burn patients, catheter-associated infections, chronic otitis media, eye infection, necrotizing pneumonia, septicemia in newborns and old debilitated persons, cystic fibrosis, malignancy, immunosuppression, and so on. ${ }^{[1]}$ It rarely causes antibiotic-associated diarrhea, ${ }^{[2]}$ however, it can cause diarrhea in immunocompromised patients $^{[3]}$ and is also associated with neutropenic enterocolitis in patients with acute leukemia, lymphoma, aplastic anemia, and so on. ${ }^{[4]}$ We report here a case of Pseudomonas diarrhea in a child suffering from acute lymphatic leukemia.

\section{CASE REPORT}

A one-year-three-month old female child, residing in Mumbai, was admitted in our hospital on 9 January, 2009, with complaints of high grade fever off and on, not associated with rigors, for the past one month. On examination, pallor was present along with ecchymosis and petechial rashes over the abdomen. She had hepatosplenomegaly. In the other systems, no abnormality was detected. Her hemoglobin was $5.7 \mathrm{gm} \%$, TLC 16,400/ cu.mm., with neutrophils $44 \%$, lymphocytes $50 \%$, and monocytes 6\%. Platelet count was 8,000/cu.mm. Serum protein was $7 \mathrm{mg}$ (albumin 4 and globulin 3), total bilirubin $1.1 \mathrm{mg} / \mathrm{dl}$ with direct bilirubin of $0.1 \mathrm{mg} / \mathrm{dl}$. ALT 31,
AST 13, and alkaline phosphatase was $337 \mathrm{IU} / 1$, serum BUN $7 \mathrm{mg} \%$, serum creatinine $0.7 \mathrm{mg} \%$, serum sodium $138 \mathrm{mEq} / 1$, potassium $4.1 \mathrm{mEq} / 1$, calcium $8.9 \mathrm{mg} \%$, and phosphate $4 \mathrm{mg} \%$. She was diagnosed as a case of severe anemia with hepatosplenomegaly and acute lymphatic leukemia - CALLA positive. She was given chloroquine on admission and later two bottles of platelets were transfused. Antibiotics (Amikacin and PiperacillinTazobactam), Prednisolone (10 mg), Vincristine $(0.36 \mathrm{mg})$, and Methotrexate ( $8 \mathrm{mg}$ ) were started. On 19 January, her hemoglobin increased to $7.7 \mathrm{gm} \%$, TLC reduced to $7600 /$ cu.mm, with neutrophils $27 \%$, lymphocytes $68 \%$, and monocytes $5 \%$. The platelet count increased to $22,000 /$ cu.mm. Two more bottles of platelet transfusion was given and the other medicines were continued.

On 27 January, she suddenly developed loose motions without abdominal pain. Stool was sent for culture along with blood culture in trypticase soy broth. After 24 hours, from the blood culture bottle, subcultures were peformed on MacConkey agar (MA) and blood agar (BA) plates and incubated at $37^{\circ} \mathrm{C}$ overnight. The stool was directly plated on MA and Xylose lysine deoxycholate agar (XLD). Pure non-lactose fermenting colonies with irregular margins grew on both the MA plates. They were oxidase positive and motile, and were identified as Pseudomonas aeruginosa by standard biochemical tests. ${ }^{[5]}$ Thus the same organism was isolated from the blood and stool cultures. Antibiotic susceptibility was carried out using the Kirby-Bauer disk diffusion method on a Mueller Hinton agar, according to 
CLSI guidelines. ${ }^{[6]}$ It was susceptible to amikacin, ofloxacin, piperacillin, and imipenem, and resistant to ceftazidime. The stool isolate, in addition, was susceptible to norfloxacin and resistant to nalidixic acid. On 27 January her complete blood count was by Hb 10.1 gm/dl, TLC 1100/cu.mm., P2, L8, Blasts90, Platelets 7,000/cu.mm.

She developed left facial palsy on 28 January and also developed perianal and gluteal abscesses.

On 29 January, she was put on amikacin, meropenem and metronidazole. Although the diarrhea subsided after five days, the stool cultures repeatedly grew P.aeruginosa. On 2 February, two stool samples were again sent for culture and both grew Psendomonas aeruginosa in pure culture, with the same antibiotic susceptibility pattern, but the blood culture sent on the same day did not show any growth. On 12 February also, the stool culture grew Pseudomonas aeruginosa, susceptible only to amikacin. Blood cultures were further sent on 9, 16, and 20 February and 2 March. None of them showed any growth. Stool cultures were further sent on 20 February, 2 and 5 March, and they did not grow any pathogenic bacteria. She was given another bottle of platelet transfusion and was discharged on 9 March. At discharge, her condition was stable.

\section{DISCUSSION}

Pseudomonas aeruginosa as a cause of infectious diarrhea is rare. ${ }^{[3]}$ It usually represents a nosocomial infection in an immunocompromised host. ${ }^{[3]}$ In PubMed Search with 'Psendomonas diarrhea in acute lymphatic leukemia', no item was found. This child was already on chemotherapy. Mucosal damage is a major risk factor for complication of cytotoxic chemotherapy. Mucosal barrier injury in the gastrointestinal tract or gut mucosal ulcerations may result from direct drug-related cytotoxicity or from neutropenia itself. $^{[7]}$

The normal fecal microflora changes in chemotherapyinduced diarrhea, in many patients, show a decreased proportion of anaerobic bacteria with a higher proportion of aerobic and oxygen-tolerant bacteria. ${ }^{[8]}$ This child was already on chemotherapy when the diarrhea developed and she was already diagnosed to be suffering from acute lymphatic leukemia. It was probable that the normally reduced nature of the lumen became oxidized following chemotherapy, inhibiting anaerobic bacteria and allowing the growth of oxygen-tolerant bacteria.

More than $40 \%$ of P.aeruginosa had the ability to colonize in the human intestine and cytotoxic activity had been demonstrated in $>80 \%$ of Pseudomonas aeruginosa isolates. ${ }^{[8]}$ Some of the isolates were weakly enterotoxigenic in infant mice and few also induced symptoms and signs of enteritis in clindamycin pre-treated rats. ${ }^{[9]}$

Neutropenic enterocolitis (NE) is a fulminant necrotizing process involving segments of the large and small intestine that occurs in the setting of agranulocytosis, commonly seen in patients with hematological malignancies, especially acute leukemia. ${ }^{[4]}$ Agarwal et al. have reported E.coli and Klebsiella from the stool culture of a leukemic child, who developed NE ${ }^{[4]}$ The present case grew Pseudomonas aeruginosa from the stool as well as blood culture in a leukemic child. Although fever and diarrhea were present in this case, abdominal pain was absent, which was one of the hallmarks of NE. Pseudomonas was also reported to cause anorectal necrotizing ulceration in cases of acute leukemia. ${ }^{[10]}$

Therefore, stool specimens of all leukemic patients suffering from diarrhea, and on chemotherapy, should be sent routinely for culture, so as to find out the exact cause of diarrhea. Apart from stool culture, blood culture is also recommended in such cases. A high index of suspicion is needed for all patients who present with fever and abdominal pain in the setting of neutropenia. ${ }^{[7]}$ Proper reporting will enable the clinicians to start appropriate antibiotics, thereby reducing the morbidity and mortality of leukemic patients, as was observed in this case.

\section{REFERENCES}

1. Chakraborty P. Pseudomonads and non-fermenters. $2^{\text {nd }}$ ed. A Textbook of Microbiology. Kolkata: New Central Book Agency (P) Ltd.; 2003. p. 343-51.

2. Kim SW, Peck KR, Jung SI, Kim YS, Kim S, Lee NY, Song JH. Pseudomonas aeruginosa as a potential cause of antibioticassociated diarrhea. J Korean Med Sci 2001;16:742-4.

3. Porco FV, Visconte EB. Pseudomonas aeruginosa as a cause of infectious diarrhea successfully treated with oral ciprofloxacin. Annals Pharmacother 1995;29:1122-3.

4. Agarwal BR, Sathe AS, Currimbhoy Z. Neutropenic enterocolitis with acute leukemia. Indian Pediatrics 1994;31:57-60.

5. Koneman EW, Allen SD, Janda WM, Schreckenberger PC, Winn WC Jr. The nonfermentative gram-negative bacilli. $5^{\text {th }}$ ed. Color Atlas and Textbook of Diagnostic Microbiology. Philadelphia: Lippincott; 1997. p. 253-72, 287-93, 299-304.

6. Performance standards for antimicrobial susceptibility testing. Seventeenth informational supplement. M100 - S17. Clinical Laboratory Standards Institute. 2007;27(1):p. 38-9.

7. Ullery BW, Pieracci FM, Rodney JR, Barie PS. Neutropic enterocolitis. Surg Infect (Larchmt) 2009;10:307-14.

8. Stringer A, Gibson R, Logan R, Bowen J, Yeoh A, Keefe D. Chemotherapy-induced diarrhea and changes in the fecal flora of cancer patients. Available from: http://www.mascc.org/ media/20th symposium proceeding/poster/2007 Human2.Pdf. [cited in 2009].

9. Adlard PA, Kirov SM, Sanderson K, Cox GE. Pseudomonas aeruginosa as a cause of infectious diarrhea. Epidemiol Infect 1998;121:237-41.

10. Givler RL. Necrotizing anorectal lesions associated with Pseudomonas infection in leukemia. Dis Colon Rectum 2007;12:438-40.

Source of Support: Nil, Conflict of Interest: None declared. 